\title{
Milliliter per Minute per 1.73 m2 of Body Surface Area
}

National Cancer Institute

\section{Source}

National Cancer Institute. Milliliter per Minute per $1.73 \mathrm{~m} 2$ of Body Surface Area. NCI

Thesaurus. Code C67412.

A unit used in the calculation of a rate of the substance removal from the body (such as creatinine clearance) for the measurement of renal excretory function, with the applied correction factor for body size. It is also used as a dose delivery rate calculation unit equal to the rate, at which one milliliter of medication is delivered during a period of time equal to one minute per 1.73 square meters of body surface area. 